\title{
Significance of Strategic Outsourcing in IT Project Management
}

This paper was downloaded from TechRxiv (https://www.techrxiv.org).

\section{LICENSE}

CC BY 4.0

SUBMISSION DATE / POSTED DATE

$26-12-2021$ / 29-12-2021

CITATION

Ravi, Akash; Donawa, Nazim (2021): Significance of Strategic Outsourcing in IT Project Management. TechRxiv. Preprint. https://doi.org/10.36227/techrxiv.17693825.v1

$\mathrm{DOI}$ 


\section{Significance of Strategic Outsourcing in IT Project Management}

Submission for CNIT 55200-001 - Fall 2021

\author{
Akash Ravi \\ Dept of Computer and Information Technology \\ Purdue University \\ West Lafayette, USA \\ ravi48@purdue.edu
}

\author{
Nazim Donawa $\mathrm{Jr}$ \\ Dept of Computer and Information Technology \\ Purdue University \\ West Lafayette, USA \\ ndonawa@purdue.edu
}

\begin{abstract}
Outsourcing refers to the concept of delegating certain tasks to an external team. It can be a differentiating factor that can aid an organization gain a competitive advantage. Consultants can bring valuable experience on domain knowledge, interdisciplinary applications, and benefits from economies of scale. From ensuring effective use of resources to venturing into uncharted sectors, outsourcing can have a myriad of benefits for a project team. Similar to most business processes, there is an inherent trade-off that needs to be made when outsourcing. Risk management protocols are required when outsourcing, to balance execution control and collaborate effectively. Within the scope of IT projects, this research aims to survey the various methods of strategic outsourcing and the contexts in which they operate. Further sections attempt to analyze case studies based on the aforementioned aspects. Based on the available literature, the research consolidates best practices in outsourcing and presents recommendations on aligning them with project goals. Finally, the potential concerns that need to be addressed when trying to outsource activities are presented. Therefore, the research to be done within this analysis will look to dissect existing literature to assess current outsourcing practices. Additionally, the research will look for reasons why outsourcing was/is not as successful as it could be, as well as evaluate how outsourcing in IT has changed with the occurrence of the COVID-19 pandemic.
\end{abstract}

Index Terms-Outsourcing, Project Management, Consultancy, Vendor Management, Contractors

\section{INTRODUCTION}

IT outsourcing is the practice of using external resources to accomplish information technology functions. The procured service or product could be used to add value to a process or a product and hence, contribute to the overall project objectives. Organizations usually consider outsourcing when they believed that certain functions can be completed faster, cheaper, or more efficiently by an outside organization. With the ways in which industries have been evolving, outsourcing has become a business imperative. However, just like any other business decision, outsourcing, can often seem like a double-edged sword. While there are numerous advantages, outsourcing methodologies can also increase the risks associated with the process. With a proper understanding of the nuances involved in outsourcing, it might be possible to use this to gain a competitive advantage and succeed. Several firms have embarked on strategizing outsourcing over the years and devised management tools to leverage them. The global IT outsourcing market size spans several hundreds of billions of dollars [1]. Yet, a surprisingly high number of organizations fail to utilize them effectively and have suffered impacts on the "triple constraints" (Scope, Time and Cost) and consequently, the overall quality of the project deliverable [2].

Outsourcing is leveraged by organizations of all sizes. For startups and small ventures, outsourcing IT can serve as the key to propelling their business faster and cheaper, when compared to what they could with a small in-house team. Consultancies can bring their expertise and experience to build robust solutions for clients who may not have all the required resources themselves. Big firms and conglomerates also tend to outsource parts of their work as it lets them focus on their core capabilities and business. While outsourcing is practiced across domains, this research focuses on IT outsourcing. The scope of modern IT consulting extends beyond traditional application development and maintenance to include digital experience, data analytics, technical innovation, risk assurance, lean operations, and business process management.

To comprehend the scale at which IT outsourcing is practiced and relevant, it can be advantageous to analyze the market prospects of the players in this sector. Some of the globally recognized technology consulting firms are Infosys, Accenture, Cognizant Technology Solutions, Capgemini, Deloitte Consulting, HCL Technologies, Wipro Technologies, and Tata Consultancy Services. These companies have clients across the globe and provide a myriad of services in industries such as aerospace, automotive, banking, consumer products, energy, entertainment, instrumentation, medicine, and telecommunication. As an example, Accenture had a revenue of 50 billion USD in 2021 and employed around 624,000 people worldwide [3]. These firms offer professional services spe- 
cialized in technologies such as cloud computing, big data, cybersecurity, logistics, and HR services. These multinational corporations provide end-to-end business solutions that can span across an IT product's life cycle. With the advent of emerging technologies, diverse organizations continue to digitally transform and use information systems for running their business. Since technology has become an indispensable part of their operations, outsourced IT functions are already running critical systems in this dynamic world [4]. Thus, the importance of understanding IT outsourcing is ought to grow in significance.

\section{Literature SURVEY}

There are multiple ways in which operations can be outsourced to other companies. The engagement could be long-term or disposable. The extent to which an external team is involved and owns the responsibility can also vary significantly. The IT infrastructure, the process, or even the end-to-end project can be outsourced. Different industries also tend to have certain components that are predominantly outsourced. While IT outsourcing methodologies can be distinguished based on all these factors, there is a lack of a universal classification due to the sheer number of potential variables [5].

Offshore IT outsourcing refers to the concept of contracting operations to a vendor based out of a different geographic region. Anna Legesse details the methods of governance and client-vendor relationships in such arrangements [6]. Under the static form of governance, the author differentiates the cost of complementing versus substituting. Static governance can also be viewed as formal or relational. Formal governance involves legally binding SLAs and KPIs, while relational governance involves unwritten and non-contractual controls. Based on the author's survey, the former is argued to be the optimal strategy driven by the characteristics of the specific type of arrangement. The latter is shown to be supported by costs, transactions, and contractual theories. The author then explains the dynamic governance model that formulates strategies based on the stages of the client-vendor relationship. A combination of formal and relational methods is mapped to specific organizational goals in dynamic governance. Finally, the text reviews potential factors that might hinder effecting collaboration while outsourcing IT operations. The roles of physical/cultural distances and employee status were mentioned as points that need to be focused on while defining IT artifacts that are being outsourced to offsite locations.

Multiple factors are involved when evaluating outsourcing partnerships and success. Jack Marchewka et al. [7] have compiled a comprehensive model for the same. The theoretical model en-comprises of the partnership quality, configuration, operationalization, dynamic, static, contextual, and cultural factors. There are numerous aspects under each of these concerns. As an example, information sharing and knowledge exchange are important activities that can significantly improve outsourcing relationships by reducing potential conflicts, building trust, and increasing commitment. Similarly, participation, joint action, and toplevel management support are all essential to enhance and nurture the partnership quality. Compatibility in the maturity of management and development practices is also important to ensure seamless collaboration between the partners. Despite all of these well-documented practices, $75 \%$ of managers in the US agreed that outsourcing initiatives did not fulfill their expectations. The authors note that the presence of these factors does not guarantee successful outsourcing. Other fundamental requirements and objectives such as schedule, cost, and quality can still significantly impact the overall success of the outsourced project. Business satisfaction and user satisfaction are attributed to successful outsourcing.

As IT outsourcing has matured over the years, the focus has broadened beyond cost-saving and short-term deliverables. There have been studies to comprehend the aspects of long-term sustainability in business value alignment. Rudy Hirschheim et al. [8] have researched concepts such as innovation, multi-vendor collaboration, internationalization, and corporate social responsibility in the realm of outsourcing information systems (IS). The Kodak deal in 1989 is the first notable use of IT outsourcing as a strategic tool. From being a cost-cutting tool, outsourcing has evolved to become an important part of the overall organizational strategy. Globalization also paved way for offshore arrangements. This commoditization of processes is also shown to have a significant impact on our economies. During recent times, newer forms such as "microsourcing", "crowdsourcing" and collective intelligence have grown alongside traditional practices. With all of these aspects of outsourcing in consideration, the authors have discussed themes such as innovation management, dynamic governance, multi-vendor ecosystems, social responsibility, and open platforms. The authors have articulated numerous questions regarding outsourcing. Notable ones focus on the overlapping of business functions and the role of organizational politics in influencing sourcing decisions. This framework provides recommendations to sustain business value output and address the needs of both the clients and the vendors.

An outsourcing arrangement doesn't end with the process of selecting the right vendor. Effective execution of an outsourced project might require organizations to maximize flexibility and control. An article in the Harvard Business Review illustrates various aspects in negotiating a successful outsourcing contract [9]. Clashes with IT suppliers on interpreting requirements and service levels are inevitable. The authors suggest the concept of measurable partnerships and shared goals to hedge against these uncertainties. Withholding a part of the business as a potential contract would make the contractor more cognizant about retaining prospects. Splitting operations between multiple suppliers can also create a threat of competition and hence, motivate 
vendors to deliver quality results. Another important aspect is to ensure that the internal teams are aware of the engagements and resonate with the business decisions. In-house system integrators are essential to fill in the gaps in the procured products or services and align them with the organizational goals. The authors also caution against unnecessary overlaps in such projects. All stakeholders must be in the loop while formulating strategies in order to be able to 'exploit' or make the best use of an outsourced disposition.

Although Information Technology outsourcing has progressed a long way, it still has its drawbacks and flaws as embedding outsourcing into IT projects is not always one-hundred percent successful. According to Yuwei Shi, "The business literature has not stressed the risks of IT outsourcing beyond the operation of a typical outsourcing project and outsourcing's short-term performance impact on the client firm." [10]. Additionally, it has not "reflected on outsourcing risk management beyond formal contract or interpersonal relationship building." [10]. Underneath the formal contract and interpersonal relationships lie "several clear signs of a failed outsourcing project. The most cited one is project cost overrun-or worse, an outsourcing project that results in the cost of an overall operation for the client." [10]. Overspending on outsourcing is "the most visible outsourcing risk because cost-cutting remains one of the most common reasons for IT outsourcing". [10]. One reasoning for this issue in cost overrun is that there is a lack of communication and understanding between the client and the main company. The main organization that is dealing with the client directly needs to portray to the client that utilizing outsourcing will not guarantee an incredible cost-saving. Additionally, there needs to be an understanding on the organization's executive/management side that there should not be an assumption made that outsourcing will guarantee savings in cost. While overspending is the most visible risk in IT outsourcing, "Other common signs of an outsourcing disaster include: clients' poor service delivery, increasing customer complaints, and, in extreme circumstances, business shutdown (due to an information security breach or the vendors' system or organizational failure)." [10]. If any of these issues were to arise in a project implementing outsourcing, it would be "directly harmful to an outsourcing client's shortterm market performance as measured by sales, costs, the strength of customer relationships, or market reputation." [10].

An interesting solution found that looks to solve issues in IT outsourcing were highlighted by Godwin G. Udo, who conducted an analysis on a technique called the "Analytic hierarchy process" or AHP. Essentially "AHP is a mathematically based, multi-objective decision-making tool which was introduced by Saaty (1990). It uses the pairwise comparison method to rank order alternatives of a problem that are formulated and solved in a hierarchical structure. The technique has the advantage of being simple and thorough in handling difficult real-life problems." [11]. This technique is quite interesting as it utilizes software that can aid in making real-life decisions and comparisons in IT outsourcing. "The process of AHP comprises the following steps: 1. Structure a problem with a model that shows the problem's key elements and their relationships. 2. Elicit judgments that reflect knowledge, feelings, or emotions. 3. Represent those judgments with meaningful numbers. 4. Use these numbers to calculate the priorities of the elements of the hierarchy. 5 . Synthesize these results to determine an overall outcome. 6 . Analyze sensitivity to changes in judgment." [11]. This model can be deemed as a nice approach in strategically selecting an outsourcing resource as it makes decisions based on the judgments of given criteria to come to a solution in what the best choice of vendor would be. It is noteworthy that the judgments have a sensitivity level that offers a variety of different scenarios where one aspect/requirement weighs more in importance than another.

There was an additional interesting aspect that may factor into the success of IT outsourcing which is risk management. A study conducted by researchers in Montreal proposed an analysis to show that outsourcing is indeed a risk and that ongoing active risk management is a proper approach in reducing risk while receiving the benefits that are affiliated with IT outsourcing. [12]. This study involved researchers conducting case studies within five different companies to measure and analyze their management in terms of risk, to conclude if overall risk management within an organization has the potential to affect the success in utilizing IT outsourcing as a resource. The data for this analysis was collected via interviews and on-sight observation where employees who are in control of or have an influence on decision making within each business were interviewed and decisions observed. Within the case studies, It was found that companies who have poor risk management in their general structure were not successful in reaping the benefits of outsourcing as these individuals lacked to associate little or no risk with IT outsourcing [12]. For example, if a company were to utilize outsourcing for a certain software package and agreed for 80 percent of it to be functional by a certain date for a set price, while the other 20 percent will be delivered in the future and the price to be negotiated, offers a large space for risk to exist. With this large percentage where risk can be allowed, there come potential problems such as the remaining 20 percent of the software package ending up being completed much later and more expensive than expected which in turn makes tension with the client. Another possibility could be that the software in the original 80 percent has issues in functionality upon the date set to be delivered and the project at hand has to be delayed even before the 20 percent portion has even been considered in starting. Therefore, accounting for and evaluating risks constantly in a business is essential for not just utilizing IT outsourcing, but in any major project. The study resulted in the conclusion that ongoing risk management is a potential approach to help reduce overall risk, and more specifically the risks that are associated with outsourcing. Managing risk 
in a proper fashion is important for businesses, as poor risk management can lead to the failure of a business, thus it can be claimed that continuous proper risk management is needed to efficiently reap the benefits of IT outsourcing.

\section{BEST PRACTICES IN IT OUTSOURCING}

Based on the literature survey, it can be inferred that IT outsourcing is a common strategy, yet not leveraged to its true capability. Different case studies and research findings over the years have resulted in diverse practices in IT Outsourcing. In the following section, we attempt to consolidate them and briefly describe some practices that can serve as guidelines when planning to outsource IT. By creating such a framework that companies can use to select, contract, and govern vendor relationships, it would be possible to capture new value and build strategic tactics.

\section{A. Elucidating goals and expectations}

The first step in planning to outsource is to collate all requirements and clearly identify the parts that would be outsourced. Identifying deliverables and quantifying their value at the inception of the project discussion allows organizations to focus on selecting the most appropriate vendor. It helps to set a baseline for the remaining phases of the process. The request for proposal (RFP) is supposed to describe the overall expectations in the outcome and solicit bids from vendors and consultants. A well-defined RFP would help vendors to understand the service level agreements and customize a precise Statement of Work (SOW). The vendors would also have to calculate the operation costs, study the market dynamics, and plan capacity investments [13]. Based on the submitted bids, a transparent evaluation process can promote mutual trust and concern. This early alignment of expectations encourages all stakeholders to thoughtfully plan, execute, and measure the delivered value.

To ensure that all processes are systematically managed, proper documentation needs to be maintained at all phases of the project. Right from sourcing to project closure, sufficient details must be recorded from tracking and future analysis. Audits and compliance procedures also expect granular metrics on every task and transaction. Even if individuals change, business continuity can remain unimpeded if sufficient documentation is maintained on both, the vendor and the client-side [14].

\section{B. Materializing prospects}

Contracts are essential to ensure business functioning and conduct [15]. It defines the nature of transactions and services to be rendered and outlines the relationships between the stakeholders. Apart from saving the company from legal trouble, the contractual clauses should also aim to foster innovation. All the key personnel must be identified and provided the required resources to lead their proposed duties. Since individuals may be employed 'at-will', all clauses related to disentanglement processes must be designed to accommodate unexpected changes in human resources. Cybersecurity is another overlooked aspect affecting outsourcing decisions [16]. Security protocols and risk management strategies should be established in the contracts. Finally, ownership of intellectual properties (IP) should also be mutually agreed upon before the engagement. Provisions to manage potential conflict of interests should be established upfront to minimize bias in judgments. A formal plan that addresses concerns such as change management, quality, compliance, and other factors that can lead to informed decision-making and effective administration over time. To build an organic incentive for the contractors, a shared vision model where the vendors have increased ownership over the project can be beneficial.

With offshore IT services, companies can leverage the benefits of a lower cost of resources (both in terms of labor compensation and bill of materials). However, this raises the concern of having to cover international tax laws and export controls. Different countries have different norms and regulations. The geopolitical situation must also be taken into consideration when planning such contracts. Beyond the clauses on paper, certain human factors also need to be oriented. Organizations need to be considerate about diverse working cultures and set up effective cross-border communication channels. Individuals need to be respectful about timezone differences, local holidays, and language accents. Maslow's hierarchy of needs can serve as a guide to ensure that individuals are empowered to deliver their expectations [17]. Without these fundamentals in place, it might be hard to expect the technical

\section{Measuring success and adaptive governance}

Traditionally, vendor governance has been a slow-paced, rigid, and one-size-fits-all approach. It predominantly focused on cost-cutting rather than looking forward to disruptive innovations. The management must adopt an agile mindset to measure progress and incrementally deliver more value. A cross-enterprise council might be able to dynamically align the priorities and ensure that everyone is on the same page. Process efficiency and standardization may need to be prioritized over short-term cost savings to ensure that no accidental 'time bombs' are waiting to explode further down the project. Members involved in the project must be able to easily navigate through the hierarchy while embracing brand differentiation.

According to Deloitte's Global Outsourcing Survey (GOS) in 2016, 65 percent of the surveyed organizations had failed to measure the intrinsic value of innovation delivered by their vendors [18]. Nevertheless, their analysis indicates a promising pathway for outsourcing to drive value-added benefits across multi-functional sectors. Deloitte's latest Global Shared Services and Outsourcing Survey Report in 2021 notes that an approximate figure of 65 percent of successful organizations incorporates some form of shared 
services and outsourcing in their delivery models. Cultivating effective and efficient outsourcing practices can thus bolster a win-win relationship between companies and their vendors.

Implementing a well-founded digital strategy across the front, middle and back-office environments is critical to running the business for organizations today. Practitioners recommend formulating a technology roadmap based on the understanding of operation models, technology architecture, and data assets. With a knowledge of the technology underpinning, the leadership teams must be able to orchestrate process, automation, and outsourcing. In any setting with two or more stakeholders, effective communication and collaboration are required. For outsourced projects, there needs a consensus among all the members regarding the overall project vision. Agile methodologies and the concept of continuous process improvement can aid organizations in proactively keeping a check on these aspects.

\section{COVID-19 IMPACT ON IT OUTSOURCING}

Recently within our current society, there has been the spread of a virus named SARS-CoV-2, to the point where it has become a worldwide pandemic. This occurrence of the pandemic impacted society on a high level to the point where staying at home became a requirement. Which in turn "has severely affected the world's manufacturing industry, particularly in terms of the continued increase in logistics costs that has led to an increase in business operating costs." [19]. "The outsourcing of IT is considered one of the most prospering businesses internationally. Nonetheless, with the COVID-19 crisis, a combined effect is anticipated in the field of IT outsourcing" [19]. The pandemic had a major effect on IT outsourcing as several companies have decided to employ host country candidates only. This means that countries that rely heavily on outsourcing as a means of business/income will take a major hit. An example of this occurred between two influential organizations of Australia, Telstra and Optus, and UK's Virgin Media, which had partnered for process outsourcing with India and the Philippines " [19]. The two companies "reported preferring employing candidates in host country only." [19], and "According to the United Nations report, India is considered among the 15 most impacted economies due to the coronavirus crisis" [12]. Additionally, with several individuals transitioning to remote work, this caused an increase in bandwidth use which in turn arose the issue of internet availability in these countries. With businesses resorting to in host country only for IT outsourcing, this meant that off-shore and overseas IT outsourcing became competitive in finding work, which in turn may have led to the decline or failure of overseas businesses.

COVID-19 has impacted travel quite a bit as there was a point in time where airlines, were shut down and it was not possible to fly anywhere. This aspect affected IT outsourcing as there may need to be regular visits between the 3rd party vendor and the main business. Additionally, COVID-19 heavily impacted imports and exports worldwide and resulted in shipping costs and time increase significantly which in turn may have affected off-shore/overseas IT outsourcing as it would make the service they provide more expensive to maintain in addition to longer shipping times. From travel being unavailable for an extended period of time, this resulted in overseas ties being broken and companies moving toward expanding in-house teams to complete the work.

At the start of the pandemic, "Things weren't still looking bright for IT service providers in the early days of the coronavirus pandemic." [20], as travel restrictions were in place, businesses had a decline in producing new projects, in addition to the inconsistencies in company revenues. The "global IT outsourcing sector sustained a heavy blow, reducing value by 5 percent" [20]. "However, once companies recognized the need to deliver a sustainable response to the crisis, the tables have turned again for IT suppliers. IT services' demand intensified with organizations accelerating digital adoption to bounce back and build resilience to weather future disruptions. [20]. With several companies having to adapt to the circumstances of the pandemic, this adaptation included moving several employees to a remote position, which in turn increased the demand for things like digital tools to aid in communication and collaboration in an online setting. Additionally, a "host of new customer demands spurred by the pandemic urged businesses to embrace new technology areas to offer faster, modern and more convenient experiences." [20]. At this point in the pandemic, a new market for IT outsourcing had been created in which several organizations were resorting to IT outsourcing to develop modern technology solutions in a faster more efficient manner, which can be referred to as digital transformations. It was found that "the 2020 Global Managed Services Report by NTT suggests that 45 percent of global corporations are planning to outsource more work in the next 18 months." [20]. It seems COVID-19s' original impact was not looking bright for the IT outsourcing field, however, it turns out that it created a wave of opportunity for overseas IT outsourcing companies.

As the pandemic progressed, it was seen that companies had higher demand for IT outsourcing, "but COVID-19 has forced a change in how businesses and suppliers interact" [21]. According to a survey taken, more than 60 percent of respondents said they would renegotiate their contracts to adjust elements such as pricing, scope, and mode of delivery [21], which is not an easy dynamic for suppliers to adjust and change quickly. It was shown in a survey that "Post-COVID-19, 96 percent of the surveyed companies want to accelerate transformations that are business-critical. But at the same time, 75 percent will continue to cancel or delay those projects that no longer stack up as priorities" [21]. Therefore, suppliers will have to account for and be able to shift to this new dynamic in IT outsourcing in developing a 
deeper relationship with the business. Additionally, businesses will need to become more strategic in where IT outsourcing is utilized as it is not always guaranteed that outsourcing will yield the desired results. Some steps that both businesses and suppliers can take to help alleviate this are: "1. Focus on resilience, 2. Selective partnerships, 3. Future-proof contracts, 4. Persist with transformation agendas, and 5. Bridge talent gaps." [21]. The first aspect of focusing on resilience mainly pertains to businesses as if a company's global network is damaged, businesses feel as though they are not prepared for what is to come, therefore businesses need to maintain a high reliance when it comes to company agendas while suppliers/ outsourced vendors will be utilized to bridge any gaps that may come. The next aspect of selective partnerships pertains to both businesses and suppliers. Businesses need to narrow their supplier ecosystems to build fewer yet deeper supplier partnerships, as “it's taxing, expensive and complicated to orchestrate a large supplier ecosystem, and selective - and deep - partnerships are far more productive." [21]. This means that outsourcing suppliers will have to produce quality results on time for businesses to continue utilizing their services. Additionally, it means, that suppliers will need to be more open to adjusting contract elements like date of completion, pricing, and scope with the business. The next aspect of future-proof contracts touches on the subject of companies increasing the use of outcome-based contracts where "Companies must try to change their supplier relationships to focus on outcomes, rather than inputs." [21].

A notable concept related to risks in business contracts is the Force Majeure clause. It is an agreement to cushion the impact when project failure or delay results from causes beyond the reasonable control of the affected party. In this context, the clauses define acts of God such as fire, floods, embargoes, wars, insurrections, riots, strikes, lockouts, or other labor disturbances. The clause minimizes the liability and risk to the contractors in such situations and may entitle them to an excuse for breach of contract. Contrary to insurance, the risk is not spread between parties to accommodate unfortunate scenarios. Rather, it formally defines the protocols that would take over usual operations during times of crisis. There have been considerations that the COVID-19 Outbreak could constitute a Force Majeure event [22]. IT projects are no exceptions and may need such provisions to ensure that businesses can engage in mutually supportive relationships.

With future-proof contracts in place, it deepens the understanding between both supplier and business of the expected outcome which can reduce issues down the road of an ongoing project. The next aspect of persisting with transformation agendas mainly pertains to businesses rather than suppliers. Businesses leaders must narrow and decide which digital transformation projects are of the most importance in achieving and which transformations are to be delayed or canceled. By doing this, businesses have a clear view of capability gaps that need to be filled by suppliers, and additionally, this will aid businesses in determining the type of suppliers needed to carry out the desired digital transformation. The last aspect of bridging talent gaps persists mainly to suppliers as it has been a trend for companies to bring in-house IT capabilities to help prevent the stalling of digital transformations or the possibility that the supplier has talent gaps in the area where they need to deliver the outcomes. For suppliers, this means that their employees need to be evaluated in terms of capabilities so that there are little to no gaps in talent when tackling a business's project.

In summary, the pandemic has caused negative and positive outcomes on IT outsourcing. It was expected that IT outsourcing would take some damage as a result of the pandemic however the boost in the demand for IT outsourcing was not necessarily an expected outcome of the pandemic. It is quite interesting how the outsourcing ecosystem changed due to the pandemic, as more opportunities for IT outsourcing became available, however the process of selecting vendors as well as the expectation from vendors has changed. As opposed to companies just using any relevant outsourcing company for a project, companies are now more carefully selecting vendors as the projects being carried out are increased in rigor and importance while doing away with delaying projects of less importance. With companies increasing selectivity in which vendor to use where, vendors must change their dynamics in being more cooperative in aspects such as outcomes, cost, and time of delivery with the clients they are providing work to, in order to build a reputation in the IT outsourcing field which in turn would result in more work opportunities coming to said IT outsourcing vendor. It is also noteworthy how a deeper relationship between vendor and business has influenced the success of IT outsourcing during the pandemic, meaning that there is more communication between the two and the outcomes and deliverables are parallel between them. Lack of communication between vendor and business has always persisted as an issue, however, this issue was highlighted more due to the pandemic as means of communication suffered in this time. Therefore, the pandemic has created a brand new ecosystem for IT outsourcing which may shape how IT outsourcing is utilized and carried out in the future.

\section{CONCLUSION}

This research study aimed to analyze and evaluate the widely used business technique known as outsourcing. The research done was more specifically targeted towards aspects that pertain to information technology outsourcing. In order to carry out a proper analysis of the business tactic, the research presents a review of literature that highlight the strategies that exist within implementing IT outsourcing, best practices in utilizing IT outsourcing, positive and negative aspects of utilizing IT outsourcing, and how major events, such as the pandemic have influenced IT outsourcing for the better and the worse. Additionally, there were solutions 
recommended to how some of these failures in IT outsourcing can be solved. It was found in the analysis that aspects like lack of communication between client and business, lack of communication between business and outsourcing vendor, poor risk management, and overspending are the main contributors to IT outsourcing being unsuccessful. With sufficient mitigation for these concerns, organizations can immensely benefit from strategic outsourcing and achieve feats that may not be attainable by solely relying on in-house IT teams.

\section{REFERENCES}

[1] J.-N. Lee, Y. Park, D. W. Straub, and Y. Koo, "Holistic archetypes of it outsourcing strategy: A contingency fit and configurational approach.," MIS Quarterly, vol. 43, no. 4, 2019.

[2] J. I. Agburu, N. C. Anza, and A. S. Iyortsuun, "Effect of outsourcing strategies on the performance of small and medium scale enterprises (smes)," Journal of Global Entrepreneurship Research, vol. 7, no. 1, pp. 1-34, 2017.

[3] "Q4 fy21 (ended aug. 31, 2021)," Aug 2021

[4] R. Črešnar, V. Potočan, Z. Nedelko, et al., "Speeding up the implementation of industry 4.0 with management tools: Empirical investigations in manufacturing organizations," Sensors, vol. 20, no. 12, p. 3469, 2020.

[5] S. Fedorova, "It outsourcing development trends," in SHS Web of Conferences, vol. 106, EDP Sciences, 2021.

[6] A. Legesse, "Client-vendor relationships in offshore it outsourcing," iSCHANNEL, vol. 16, no. 1, 2021.

[7] J. T. Marchewka and S. Oruganti, "A combined model of it outsourcing partnerships and success," Communications of the IIMA, vol. 13, no. 2, p. 6, 2013.

[8] R. Hirschheim and J. Dibbern, "Information technology outsourcing: Towards sustainable business value," in Information Systems Outsourcing, pp. 1-19, Springer, 2014.

[9] M. Lacity, D. Feeny, and L. Willcocks, "It outsourcing: Maximize flexibility and control," Harvard Business Review, Aug 2014.

[10] Y. Shi, "Today's solution and tomorrow's problem: The business process outsourcing risk management puzzle," California Management Review, vol. 49, no. 3, pp. 27-44, 2007.

[11] G. G. Udo, "Using analytic hierarchy process to analyze the information technology outsourcing decision," Industrial Management \& Data Systems, 2000.

[12] B. A. Aubert, M. Patry, and S. Rivard, "A framework for information technology outsourcing risk management," ACM SIGMIS Database: the DATABASE for Advances in Information Systems, vol. 36, no. 4, pp. 9$28,2005$.

[13] T. Jain, J. Hazra, and T. Cheng, "Bidding for outsourcing contracts with capacity investments and cost asymmetry," Journal of the Operational Research Society, vol. 71, no. 12, pp. 1986-2012, 2020.

[14] T. Myklebust, T. Stålhane, G. K. Hanssen, T. Wien, and B. Haugset, "Scrum, documentation and the iec 61508-3: 2010 software standard," in International Conference on Probabilistic Safety Assesment and Management (PSAM). PSAM, Hawaii, 2014.

[15] D. Fitoussi and V. Gurbaxani, "It outsourcing contracts and performance measurement," Information Systems Research, vol. 23, no. 1, pp. 129143, 2012.

[16] M. Benaroch, "Cybersecurity risk in it outsourcing—challenges and emerging realities," in Information Systems Outsourcing, pp. 313-334, Springer, 2020.

[17] P. V. Jenster and H. S. Pedersen, "Deal maker or deal breaker: human resources issues in successful outsourcing projects," Strategic change, vol. 8, no. 5, pp. 263-268, 1999.

[18] D. Smith, "Information technology outsourcing (ito) zoom in on value," Deloitte United States, Aug 2020.

[19] L. Ntasis, K. Koronios, and T. Pappas, "The impact of covid-19 on the technology sector: The case of tata consultancy services," Strategic Change, vol. 30, no. 2, pp. 137-144, 2021.

[20] R. Krajewski, "Council post: Why the pandemic led to an increase in it outsourcing," Forbes, Jan 2021.
[21] H. H. Heiner Himmelreich, "Reimagining outsourcing after covid-19," ComputerWeekly.com, Mar 2021.

[22] C. B. Casady and D. Baxter, "Pandemics, public-private partnerships (ppps), and force majeure- covid-19 expectations and implications," Construction Management and Economics, vol. 38, no. 12, pp. 1077$1085,2020$. 